\title{
Research advance in the diagnosis of pancreas divisum
}

\author{
Zongfu Zheng ${ }^{1}$, Qicai Liü,3* \\ ${ }^{1}$ Department of Laboratory Medicine, the 476 Hospital, Fuzhou, China; \\ ${ }^{2}$ Department of Laboratory Medicine, the first Affiliated Hospital, Fujian Medical University, Fuzhou, China; \\ ${ }^{3}$ Department of Gene Dignosis, Fujian Medical University, Fuzhou, China; \\ *Corresponding Author: lqc673@yahoo.com.cn
}

Received 10 August 2010; revised 24 August 2010; accepted 2 September 2010

\begin{abstract}
Pancreas divisum is a kind of congenital anatomic abnormality; its diagnostic basis depends mainly on imaging examination. With the development of medical science, imaging technology has been improved and added, and a complete examination system including ERCP, B-Ultrasound, MRCP, S-MRCP and CT, etc. has been formed. There are even researcher, through further analysis of pancreas divisum on the level of genes, found that CFTR is a risk factor causing such disease. This paper is focused on the value of these examination methods in the diagnosis of pancreas divisum.
\end{abstract}

Keywords: Pancreas Divisum; Endoscopic Retrograde Cholangiopancreatography (EPCR); Magnetic Resonance Cholangiopancreatography (MRCP); Computed Tomography (CT); Cystic Fibrosis Transmembrane Conductance Regulator (CFTR)

\section{SUMMARY}

Pancreas divisum originally referred to the double-pan creas formed as the dorsal pancreas cannot be fused with the ventral pancreas, a rare congenital malformation. While in nowadays its definition is broadened to be a kind of congenital dysplasia, the most common congenital variation in the development process of pancreatic duct, and usually refers to the infusion of ventral pancreatic duct and dorsal pancreatic duct in the process of development [1]. Initially, researchers found that the probability of PD among idiopathic pancreatitis patients is higher. Cotton [2] once reported that it is found that there were $3.6 \%$ of 169 patients who took pancreatography examination because of biliary tract diseases have got pancreas divisum; in addition, there is $25.6 \%$ of idi- opathic pancreatitis patients have got pancreas divisum found through pancreatography examination [3]. And someone thought that PD may occur at any age and the incidence of PD among crowds is at a rate of about 10\% [4]. The clinical manifestations of PD, a congenital anatomic abnormality, include non-symptom, relapsing pancreatitis, chronic pancreatitis and severe acute pancreatitis, and its diagnostic methods rely mainly on the imaging examination technologies such as ERCP, BUltrasound, MRCP, S-MRCP and CT, etc. Through research on the level of genes, it is found that CFTR is a risk factor causing PD. This paper will discuss the research progress of these diagnostic methods of PD.

\section{DIAGNOSTIC APPLICATION OF EN- DOSCOPIC RETROGRADE CHOLANGIOPANCREATOGRAPHY (EPCR) IN PANCREAS DIVISUM}

Although PD has been recognized by anatomist a few hundred years ago, it was not until 1970, with the emergence of ERCP, there were some researchers proposed that pancreas divisum and pancreatitis are associated with each other. People often think that relatively narrow occurs as PD patiets draining pancreatic juice through dorsal pancreatic duct and minor papilla, and thus led to the occurrence of pancreatitis. Until now, however, the relationship between pancreas divisum and pancreatic diseases is not yet clear. The incidence of pancreatic diseases among pancreas divisum patients does not has a significant increase compared with that of normal people. However, studies show that a large number of pancreas divisum patients can indeed progressed to pancreatic diseases. As a result, the clinical value of this link is particularly important. Endoscopic retrograde cholangiopancreatography (EPCR) can provide clear and direct image of pancreatic duct; and with a higher rate of correct diagnosis of lesions in biliary cavity and wall, it can 
clearly display the size, number, narrow scope and position of the lesions. And for papilla lesion, it can carry out biopsy, this technology is one of the main means of diagnosing pancreas and bile duct disease at present. EPCR presents a significant role in the diagnosis of PD [6-10]. Freeman ML, Nelson DB [11], and some others believe that the uppermost danger of ERCP is the complications of acute pancreatitis, the incidence of which is up to $20 \%$ among SOD patients and also $5 \%-6 \%$ among general population. For this reason, in the past 10 years, ERCP is replaced by endoscopic ultrasound (EUS) in diagnosing biliary calculi, tumor, pancreas divisum and pancreatic cyst. As EUS is characterized by high accuracy and high security [12-15], and the same, it also has limitations as can not make clear diagnosis of SOD (Sphincter of Oddi Dysfunction). The research of Coyle et al [16] shows while take the diagnostic application of ERCP in SOM (sphincter of Oddi manometry) and bile analysis, there are $80 \%$ of the patient can be diagnosed, of which $20 \%$ are diagnosed as PD; in comparation, EUS is quite useful in diagnosing biliary diseases and monitoring tumors, it can diagnose that there are 9 cases of patients suffering from chronic pancreatitis while ERCP cannot do. But EUS cannot diagnose any patients with SOD. Compared with EUS, one of the main advantages of ERCP is its ablility to monitor and show the pressure, and once required interventional therapy can be taken to make diagnosis and treatment, without the need for such traumatic diagnosis means as sphincterotomy [17]. Napoleon [18] and some others think that EUS diagnosis can be made to replace ERCP in diagnosis of common bile duct stones.

\section{DIAGNOSTIC APPLICATION OF MAGNETIC RESONANCE CHOLAN- GIOPANCREATOGRAPHY (MRCP) IN PANCREAS DIVISUM}

MRCP is an up-to-date technology for the observation of the systematic anatomy and pathology forms of pancreatic duct. The anatomy and lesions of pancreaticobiliary duct system could be shown perfectly in physical circumstances with it. MRCP is safe, non invasive, has got no contraindications and complications. In the experiment on assessing outflow obstruction in pancreatic duct with primary acute recurrent pancreatitis, Khalid et al [19] apply MRCP of pancreatic exocrine response to secretin to 10 patients with primary acute recurrent pancreatitis, followed by EPCR, divided into two groups of with manometry and without manometry. The MRCP finding pancreatic exocrine response to secretin can provide high-quality imaging and be able to make diagnosis of high specificity to pancreatic duct obstruction by us- ing pressure gauge measurement standard and clinical standard. All these illustrate the advantages of MRCP in observing pancreatic duct abnormality and diagnosing PD. The coincide rate of ERCP and MRCP is $83 \%-100 \%$ for lumen expansion, 70\%-92\% for lumen stenosis and is $92 \%-100 \%$ for filling defect [20]. In evaluation of the common diseases of the pancreas, MRCP is a nontraumatic substitute method for ERCP, and can make sure that there is no complication in the anatomical structure of the lumen. For the determination of common anatomic variation, conditions of pancreatic duct with pancreatitis, MRCP is quite valuable, especially combined with MRI imaging. However, MRCP has its limitations: 1) all patients not suitable for MRI (such as the patients wear cardiac pacemakers) are not suitable for applying MRCP; 2) severe bile-pancreas duct stenosis often manifested as the signal interruption of certain section, it cannot find the lesions accomponied with stenosis; 3) the images after reconstructed with maximum intensity projection reconstruction technology is easy to cover up minimal lesions. Ueno, etc. [21] also have reported the MRCP's difficulty in displaying short, thin ventral pancreatic duct while it is relatively easy to show thicker ventral pancreatic with a length over $2.8 \mathrm{~cm}$. In recent years, the researchers also suggested that secretin-enhanced dynamic CPCR (S-MRCP) can clearly show the infused dorsal and ventral pancreatic ducts of PD patients, and so as to enhance the image quality of the pancreatic duct, reduce the false negative rate in MRCP diagnosis, as well as improve the detection rate [22-26]. Secretin-enhanced ultrasound examination is also a non-invasive examination method, which could get consecutive ultrasonic images of pancreatic duct. But due to the limitation of the patiets' somatotype and the gas inside the site, it cannot show very clearly anatomy abnormality of pancreas divisum.

\section{DIAGNOSTIC APPLICATION OF CT IN PANCREAS DIVISUM}

Computed tomography (CT) is usually used to analyze the pancreas size, outline and focal lesions of patients with chronic pancreatitis or chronic abdominal pain. It is much more difficult for CT to show pancreatic duct structures than showing substantial pancreatic structure. By using three-dimensional reconstruction thin -slice spiral CT can get 3-D images of high quality, the diagnostic criteria of which is the infuse of the dorsal and ventral pancreatic duct and the direct connection of dorsal pancreatic duct and common bile duct. As conventional CT cannot display the duct system of the entire pancreas, conventional CT has little action in diagnosis of PD [27]. It is feasible for the multi-detect row computed tomography (MDCT) to take assessment of PD 
when the pancreatic duct can be seen clearly. With MDCT, Soto JA, Lucey et al [28] found 10 PD patients among 73 patients with pancreatic diseases, in comparison, there are $9 \mathrm{PD}$ patients found with ERCP. In addition, there is 1 false positive case included in experimental group 1 with MDCT while there are 2 in group 2 . Therefore, experimental group 1 shows that in the diagnosis of PD, MDCT's sensitive rate is 95\% and its specificity is 95\%; experimental group 2 shows that in the diagnosis of PD, MDCT's sensitive rate is $95 \%$ and its specificity is $97 \%$. Therefore, MDCT is very valuable in the diagnosis of PD, subject to the pancreatic duct is clearly visible.

\section{APPLICATION OF GENE DETECTION IN THE DIAGNOSIS OF PD}

Dray X [29] and someone else studied the relationship between Cystic Fibrosis Transmembrane Conductance Regulator (CFTR) and recurrent pancreatitis caused by pancreas divisum. Their results suggest that even mild decline of CFTR function can lead to significant changes in pancreatic exocrine function, that is, to arise the increases of the viscosity of exocrine of pancreas and the formation of protein precipitation through the secretion of images chloride and bicarbonate, and the function decrease of this gene may also result in excessive inflammatory response and thus causing local tissue adema. Taken together, CFTR dysfunction leads to the occurrence of pancreatitis through the duct and inflammatory edema caused by. They also pointed out that the occurrence of pancreatitis caused directly by itself is accidental, but when combined with CFTR dysfunction, pancreas divisum becomes an important factor causing pancreatitis. CFTR dysfunction itself is not an important factor for the onset of pancreatitis, but when combined with modifier gene abnormality, alcohol consumption or and other congenital anomalies, it may cause pancreatitis. It is very important to know pancreas divisum, a kind of congenital abnormality, since its existing ratio is relatively high among the population. Although the possibility of pancreatic diseases being caused directly by CFTR itself is low, the CFTR abnormality could serve as a foundation for pancreatic diseases. The abnormality of CFTR often leads to pancreatitis increasing the density of pancreatin, which in turn gives rise to or be accompanied by local inflammation, making the accessory nipple narrow, and finally results in total and relative blockage of major drainage pancreatic ducts, and thus brings about pancreatic disease symptoms, namely pancreas divisum [29]. In other words, CFTR is a dangerous factor contributing to pancreas divisum, so it can be used as an auxiliary diagnosis standard for assessing the pancreas divisum.

\section{CONCLUSION}

Pancreas divisum, the most common congenital mutation in the development of pancreatic duct, was not recognised until the 1970s when EPCR was invented and applied. EPCR is the golden criteria for diagnosing pancreas divisum, and also a main treatment for it. In recent years, the rapid development of the photography technology has improved the role of technologies such as Ultrasound, Thin-slice CT, MDCT, MRCP in diagnosing pancreas divisum. Especially, the present S-MRCP with noninvasive and highspecific characteristics is the most promising diagnostic means [30]. The above-mentioned dianostic means have their own limitations in clinical application. The gene diagnosis also supplies the patients suffering pancreas divisum with another choice. And as an alterative method in diagnosis and research, it leads us to an in-depth understanding of pancreas divisum at molecular level.

\section{REFERENCES}

[1] Kozu T., Suda, K. and TokI, F. (1995) Pancreatic development and anatomical variation. Gastrointestinal Endoscopy Clinics of North America, 5, 1.

[2] Cotton, Pb. (1980) Congenital anomaly of pancreas divisum as cause of obstructive pain and pancreatitis. Gut, 21, 105.

[3] Sklein, J. (2004) Pancreas divisum, an evidence-based review: part I. Gastrointestinal Endoscopy, 60, 419.

[4] Varshney, S. and Johnson, C.D. (1995) Pancreas divisum. International Journal of Pancreatol, 25, 135-141.

[5] Staritzm, M. and Buschenfelde K. (1988) Elevated pressure in the dorsal part of pancreas divisum: The cause of chronic pancreatitis? Pancreas, 3, 108.

[6] Coyle, W.j., Pineau, Bc., Tarnasky, Pr., Knapple, Wl., Aabakken, l., Hoffman, Bj., Cunningham, J.t., Hawes, R.h. and Cotton, Pb. (2002) Evaluation of unexplained acute and acute recurrent pancreatitis using endoscopic retrograde cholangiopancreatography, sphincter of Oddi manometry and endoscopic ultrasound. Endoscopy, 34, 617-623.

[7] Kinney, Tp. and Treeman, Ml. (2005) The role of endoscopic retrograde cholangiopancreatography and endoscopic ultrasound in diagnosis and treatment of acute pancreatitis. Minerva Gastroenterol Dietol, 51, 265-288.

[8] Canlas, Kr. and Branch, Ms. (2007) Role of endoscopic retrograde cholangiopancreatography in acute pancreatitis. World Journal of Gastroenterol, 13, 6314-6320.

[9] Kinney, Tp., lai, R. and Freeman, Ml. (2006) Endoscopic approach to acute pancreatitis. Rev Gastroenterol Disord, 6, 119-135.

[10] Liu, Q.C., Gao, F., Ou, Q.S., et al. (2008) Novel mutation and polymorphism of PRSS1 gene in the Chinese patients with heredity pancreatitis and chronic pancreatitis. Chinese Medical Journal, 121, 108-111.

[11] Freeman, M.L., Nelson, D.B., Sherman, S., Haber, G.B., Herman, M.E., Dorsher, P.J., Moore, J.P., Fennerty, M.B., 
Lande, J.D. and Pheley, A.M. (1996) Complications of endoscopic biliary sphincterotomy. New England Journal of Medicine, 335, 9.

[12] Petrone, M.C., Arcidiacono, P.G., Testoni and P.A. (2008) Endoscopic ultrasonography for evaluating patients with recurrent pancreatitis, World Journal of Gastroenterol, 14, 1016-1022.

[13] Kinney, T.P., Lai, R. and Freeman, M.L. (2006) Endoscopic approach to acute pancreatitis. Rev Gastroenterol Disord, 6, 119-135.

[14] Tandon, M. and Topazian, M. (2001) Endoscopic ultrasound in idiopathic acute pancreatic. American Journal of Gastroenterol, 96, 705-709.

[15] Rizk, M.K. and Gerke, H. (2007) Utility of endoscopic ultrasound in pancreatitis: A review. World Journal of Gastroenterol, 13, 6321-6326.

[16] Coyle, W.J., Pineau, B.C., Tarnasky, P.R., Knapple, W.L., Aabakken, L., Hoffman, B.J., Cunningham, J.T. and Hawes, R. (2002) Evaluation of unexplained acute recurrent pancreatitis using endoscopic retrograde cholangiopancreatography, sphincter of Oddi manometry and endoscopic ultrasound. Endoscopy, 34, 617-623.

[17] Mohammad, A1.M. and Wallace, B. (2008) Diagnostic approach to patients with acute idiopathic and recurrent pancreatitis, what should we done? World Journal of Gastroenterol, 14, 1001-1010.

[18] Napoleon, B., Dumortier, J., Keriven-Souquet, O., Pujol, B., Ponchon, T. and Souquet, J.C. (2003) Do normal findings at biliary endoscopic ultrasonography obviate the need for endoscopic retrograde cholangiography in patients with suspicion of common bile duct stone? A prospective follow-up study of 238 patients. Endoscopy, 35, 411-415.

[19] Khalid, A., Peterson, M. and Slivka, A. (2003) Secretinstimulated magnetic resonance pancreaticogram to assess pancreatic duct outflow obstruction in evaluation of idiopathic acute recurrent pancreatitis: A pilot study. Digestive Diseases and Sciences, 48, 1475-1481.

[20] Hellund, J.C., Geitung, J.T., Meo, A.M., et al. (2002) Secretin stimulated magnetic resonance cholangiopancreatography in diseases of biliary and pancreatic ducts. Tidsskr Nor Laegeforen, 122, 691-694.

[21] Ueno, E., Takada, Y., Yoshida, I., Joda, J., Sugiura, T. and Toki, F. (1998) Pancreatic diseases: evaluation with MR cholangiopancreatofraphy. Pancreas, 16, 418-426.

[22] Mariani, A., Curioni, S., Zanello, A., Passaretti, S., Masci, E., Rossi, M., Del Maschio, A. and Testoni, P.A. (2003) Secretin MRCP and endoscopic pancreatic manometry in the evaluation of sphincter of Oddi function: a comparative pilot study in patients with idiopathic recurrent pancreatitis. Gastrointestinal Endoscopy, 58, 847-852.

[23] Testoni, P.A., Mariani, A., Curioni, S., Zanello, A. and Masci, E. (2008) MRCP-secretin test-guided management of idiopathic recurrent pancreatitis: long-term outcomes. Gastrointestinal Endoscopy, 67, 1028-1034.

[24] Czakó, L., Takács, T., Morvay, Z., Csernay, L. and Lonovics, J. (2004) Diagnostic value of secretin-enhanced magnetic resonance cholangiopancreatography (S-MRCP) after unsuccessful endoscopic retrograde cholangiopancreatography. Orv Hetil, 145, 529-534.

[25] Sai, J.K., Suyama, M., Kubokawa, Y. and Watanabe, S. (2008) Diagnosis of mild chronic pancreatitis (Cambridge classification): Comparative study using secretin injection-magnetic resonance cholangiopancreatography and endoscopic retrograde pancreatography. World Journal of Gastroenterol, 14, 1218-1221.

[26] Manfredir, R., Costamagna, G., Brizimg, M.G., et al. (2000) Severe chronic pancreatitis versus suspected pancreatic disease dynamic MR cholangiopancreatography after secretin- stimulation. Radiology, 214, 849.

[27] Liu, Q.C., Gao, F., Zhuang, Z.H., et al. (2007) Multisite Heterozygous mutations of PRSS1 Gene and Clinical Characterization of Patients with Hereditary Pancreatitis in the Chinese. Progress in Biochemistry and Biophysics, 34, 1269-1278.

[28] Soto, J.A., Lucey, B.C., Stuhlfauct and J.W. (2005) Pancreas divisum: Depiction with multi-detector row CT. Radiology, 235, 503-508.

[29] Dray, X., Fajac, I., Bienvenu, T., Chryssostalis, A., Sogni, P. and Hubert, D. (2007) Association of pancreas divisum and recurrent acute pancreatitis with the IVS8-5T-12TG allele of the CFTR gene and CFTR dysfunction. Pancreas, 35, 90-93.

[30] Kamisawa, T., Tu, Y., Egawa, N., Tsurutak, K., Okamoto A and Kamata, N. (2007) MRCP of congential pancreaticobiliary malformation. Abdom Imaging, 32, 129- 133. 